\title{
Delay-constrained maximum reliability routing over lossy links
}

\author{
Zhenhua Zou, Pablo Soldati, Haibo Zhang and Mikael Johansson
}

\begin{abstract}
This paper studies the problem of joint routing and transmission scheduling for reliable real-time communication over lossy networks. We impose a strict latency bound on the packet delivery from source to destination and develop transmission scheduling policies that maximize the probability that the packet is delivered within the specified deadline. A solution to this problem allows to characterize the set of achievable latencies and packet loss probabilities for a given network. We develop dynamic programming-based solutions for deadlineconstrained maximum reliability routing under Bernoulli and Gilbert-Elliot packet loss models. Particular instances of the problem that admit numerically efficient solutions are discussed and our results are demonstrated on several examples.
\end{abstract}

\section{INTRODUCTION}

Low-power wireless technologies now offer the opportunity to migrate traditionally wired automation infrastructure to flexible wireless networks. While a large research effort has been devoted to developing networked control algorithms that are robust to network deficiencies such as latency and loss, relatively little has been done on optimizing network resources to provide timely and reliable communications. Some notable exceptions include [1], [2], [3].

Unlike wired networks, wireless multi-hop networks are notorious for high loss rates and/or long packet latencies. The quality of a wireless link varies over time due to several factors, including natural channel variations and external interference [4]. Although erasure events on links can be considered independent in some scenarios (e.g. static networks with long inter-packet transmission times), packet losses are typically correlated in time [3], [5]. Still, most existing routing schemes use metrics based on average channel quality (such as the ETX metric [6]) and fail to capture channel burstiness. If packets are routed over a bursty link which happens to be in bad state, the packet will suffer a long delay waiting for the channel to recover. This has a negative impact not only on the packet latency, but also on deadlineconstrained reliability (since there will be fewer opportunities for retransmissions on the remaining path to the destination). To improve routing performance, channel burstiness must be taken into account. Recent research in this direction includes single-path routing protocols with link metrics based on the maximum burst length [3] and multi-path routing protocols that exploit bursty links in good state [5]. Several recent and emerging standards, such as WirelessHART [7], ISA100 [8] and IETF Roll [9], support multi-path routing for enhancing

\footnotetext{
This work was sponsored in part by the European Commission project WIDE, the Swedish Agency for Innovation Systems project reSENSE and the Swedish Foundation for Strategic Research project Promos.

The authors are with the School of Electrical Engineering, KTH, Sweden. Email: $\{$ firstname.secondname\}@ee.kth.se
}

reliability in wireless mesh networks. However, the open literature offers little if any insight on how to optimize routing and transmission scheduling in these systems to ensure reliable real-time communications.

This paper considers the problem of joint routing and transmission scheduling for reliable real-time communication over lossy networks. By imposing a strict latency bound for the packet delivery from source to destination, we develop transmission scheduling policies aiming at maximizing the probability that the packet is delivered within the deadline. We assume a routing topology in the form of a directed acyclic graph and derive policies under two loss models including the Bernoulli model in which the loss events are independent, and the two-state Gilbert-Elliot (GE) model [10], [11] which allows to model bursty channels with correlated packet losses. We offer a complete solution to the problem based on dynamic programming techniques when erasure events on links follow a Bernoulli process and when losses can be described by the GE model with a link state estimator. We discuss some special cases without link state estimator that admit computationally efficient solutions, and demonstrate our schemes numerically on sample networks.

\section{MODEL AND PROBLEM FORMULATION}

We consider multi-hop communications over a wireless network. The network topology is represented by a graph $\mathcal{G}=(\mathcal{N}, \mathcal{L})$ where $\mathcal{N}$ is the set of nodes and the presence of a directed link $(m, n) \in \mathcal{L}$ means that node $m$ can transmit a packet to node $n$. Packets are routed along a destinationoriented directed acyclic graph (DODAG) $\mathcal{G}^{\prime}=\left(\mathcal{N}^{\prime}, \mathcal{L}^{\prime}\right)$ with $\mathcal{N}^{\prime} \subseteq \mathcal{N}$ and $\mathcal{L}^{\prime} \subseteq \mathcal{L}$ whose destination node $z$ is reachable from all nodes in $\mathcal{N}^{\prime}$. We use the notation $\mathcal{N}_{i}$ to denote the set of neighbors of node $i$ in this DODAG.

Communication is slotted, and a single time slot allows the transmission of a single packet and the associated link level acknowledgment. We assume that links are unreliable, and erasure events on links follow an underlying two-state Markov chain as in Figure 1. The Markov chains for different links are independent of each other. This model has two

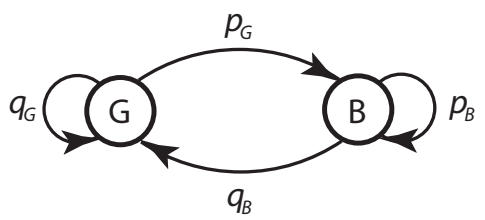

Fig. 1. Two-state Markov chain model for packet erasures over a link.

states, G (for good) and B (for bad), corresponding to successful transmission and packet loss, respectively. The 
Markov chain evolves in discrete time and state changes coincide with the time slot boundaries. Hence, the probability of successful packet transmission at time slot $t$ given that the Markov chain was in good state during time slot $t-1$ equals $q_{G}$, and the conditional probability of successful transmission at time $t$ given that the Markov chain was in bad state during time slot $t-1$ is $q_{B}$. The average packet loss probability is

$$
\pi_{B}=\frac{1-q_{G}}{1-q_{G}+q_{B}},
$$

and the unconditional probability of successful transmission is $\pi_{G}=1-\pi_{B}$. Let $T_{G}$ and $T_{B}$ be the average bust length (number of time slots) in good or bad state, respectively i.e.

$$
T_{G}=\frac{1}{1-q_{G}}, \quad T_{B}=\frac{1}{q_{B}} .
$$

The two-state Markov chain model reduces to the Bernoulli model of independent packet erasures when $p_{B}=p_{G}=p$ and $q_{B}=q_{G}=q=(1-p)$. Despite its simplicity, the GE model has proven to be reasonably accurate in capturing real packet loss behavior and the parameters $q_{G}$ and $q_{B}$ can be readily estimated from loss traces, see e.g. [4].

We consider a scenario where a single packet, generated by an arbitrary node at time $t=0$, should be transmitted over the DODAG to the sink within a deadline of $D$ time slots. Our aim is to find the joint routing and scheduling policy that maximizes the probability that the packet reaches its destination within the deadline. The optimal policy hinges on what knowledge we can assume about the state of the underlying Markov chain at the time when we make the scheduling decision. We assume that nodes can only know the states of their own outgoing links and consider three information patterns at the slot boundary from $t-1$ to $t$ :

(a) Nodes know which of their outgoing links will be in good state at the next transmission time slot $t$.

(b) Nodes know the state of their outgoing links during the previous time slot $t-1$, and hence only the conditional probability of successful transmission for the next transmission time slot $t$.

(c) Nodes do not know the state of their links until they are used. Once a link has been used, the conditional probability of successful transmission at any future time can be computed using the Markov chain.

Let us briefly comment on these information patterns. Information pattern (a) is not realistic, as it assumes that nodes can know the transmission results ahead of time. However, it provides an upper bound on the achievable reliability and gives insight into the optimal policies for the other information patterns. Also information pattern (b) can be challenged, since it assumes full state knowledge at time slot $t-1$, but it approaches a situation in which link state estimators continuously track the channel evolution. Finally, information pattern (c) is the most relevant one for a single packet transmission in a system without link state estimators. However, the optimal policy becomes quite involved, and the computing complexity of optimal policy is substantial.

\section{REliABlE ROUTING UNDER BERNOUlli LOSSES}

We first consider the case when erasure events on links are uncorrelated in time. Let $p(i, j)$ and $q(i, j)$ denote the packet loss and success probability of link $(i, j)$, respectively. Since packet losses are independent from time slot to time slot, knowing the link state at any earlier time does not influence the current routing decision. Hence, information pattern (b) and (c) will coincide and can be considered together. Next, we develop a dynamic programming approach for determining the optimal next-hop routing policy for each node with a given delay constraint, and consequently the routing that maximizes the probability of successful end-toend communication within the given deadline. Although our problem and solution are very different from the expected delay solution by Ogier [1], we use similar techniques in solving information pattern (a).

\section{A. Solution for information pattern (a)}

Consider an arbitrary node $i$ holding a packet that needs to be delivered to the sink node $z$ within $d$ time slots. Let $R_{i}(d)$ denote the probability that node $i$ will be able to complete this task, and assume that node $i$ has the knowledge of $R_{i}(d-1)$ and $R_{j}(d-1) \forall j \in \mathcal{N}_{i}$. Given an integer $k_{d} \in\left[1,\left|\mathcal{N}_{i}\right|\right]$, let $\Gamma_{k_{d}}$ denote the following transmission policy at node $i$ : introduce an ordering $\left\{j_{1}, j_{2}, \cdots, j_{\left|\mathcal{N}_{i}\right|}\right\}$ of the next-hop nodes $\mathcal{N}_{i}$ in terms of decreasing reliability

$$
R_{j_{m}}(d-1) \geq R_{j_{m+1}}(d-1), \quad \forall j_{m} \in \mathcal{N}_{i} .
$$

and forward the packet on the first (according to the ordering) link $\left(i, j_{m}\right), m \leq k_{d}$ that is in good state; if all $k_{d}$ links are in bad state, then keep the packet at node $i$ and apply policy $\Gamma_{k_{d-1}}$ at the next time slot.

Intuitively, $R_{i}(d)$ depends on $k_{d}$. If $k_{d}$ is too large, node $i$ might forward the packet to a node with low reliability. If $k_{d}$ is too small, on the other hand, node $i$ might lose the chance to forward the packet to a node with high reliability. The following theorem gives the optimal value of $k_{d}$ in terms of maximizing the success probability of delivering the packet with maximum delay constraint $d$ given the successful delivery probability of itself and its neighbors with maximum delay constraint $d-1$.

Theorem 3.1: Let $k_{d}^{\star}$ be the largest integer such that

$$
R_{j_{m}}(d-1)>R_{i}(d-1), \quad \forall m \leq k_{d}^{\star} .
$$

Then transmission policy $\Gamma_{k_{d}^{\star}}$ maximizes $R_{i}(d)$.

Proof: Given $k_{d} \neq k_{d}^{\star}$, there are two cases to consider: Case $1 k_{d}<k_{d}^{\star}$ : If all $k_{d}^{\star}$ links are in bad state, the reliability of policy $\Gamma_{k_{d}}$ and $\Gamma_{k_{d}^{\star}}$ both equal $R_{i}(d-1)$. Similarly, if one of the first $k_{d}$ links is in good state, then the policies also coincide. However, if the first link $\left(i, j_{m}\right)$ in good state satisfies $k_{d}<m \leq k_{d}^{\star}$ then the reliability of $\Gamma_{k_{d}}$ is $R_{i}(d-1)$ which, by (3), is less than the reliability $R_{j_{m}}(d-1)$ obtained by policy $\Gamma_{k_{d}^{\star}}$. Hence, policy $\Gamma_{k_{d}}$ is worse than policy $\Gamma_{k_{d}^{\star}}$. Case $2 k_{d}>k_{d}^{\star}$ : if all the first $k_{d}$ links are in bad state, or if the first link $\left(i, j_{m}\right)$ in good state satisifies $m \leq k_{d}^{\star}$, the two policies coincide. However, if the first link $m$ in good 
state satisfies $k_{d}^{\star}<m \leq k_{d}$, then $\Gamma_{k_{d}}$ activates this link and achieves reliabilty $R_{j_{m}}(d-1)$ which, by (3) is at best equal to the reliability $R_{i}(d-1)$ obtained by $\Gamma_{k_{d}^{\star}}$. Hence, $\Gamma_{k_{d}^{\star}}$ is better or equal to $\Gamma_{k_{d}}$. Since we have considered all the cases, the theorem is proved.

According to policy $\Gamma_{k_{d}}$, the probability that node $i$ forwards the packet to a node $j_{m}$ with $m \leq k_{d}$ is $q\left(i, j_{m}\right) \prod_{l=1}^{m-1} p\left(i, j_{l}\right)$, and the probability that node $i$ keeps the packet is $\prod_{m=1}^{k_{d}} p\left(i, j_{m}\right)$. With $k_{d}=k_{d}^{\star}$, we have

$$
\begin{aligned}
R_{i}(d)= & \sum_{m=1}^{k_{d}^{\star}} R_{j_{m}}(d-1) q\left(i, j_{m}\right) \prod_{l=1}^{m-1} p\left(i, j_{l}\right) \\
& +R_{i}(d-1) \prod_{m=1}^{k_{d}^{\star}} p\left(i, j_{m}\right)
\end{aligned}
$$

Then, for any $i \in \mathcal{N}^{\prime}$ and a specified deadline $D, k_{D}^{\star}$ and $R_{i}(D)$ can be efficiently calculated by applying Theorem 3.1 and Eq. (4) recursively using dynamic programming. Here, we present a distributed scheme to solve the problem in $D$ steps. The algorithm is initialized as follows:

$$
\begin{gathered}
R_{z}(d)=1, \quad \forall d=[0, D] ; \\
R_{i}(0)=0, \quad \forall i \neq z .
\end{gathered}
$$

In Step 1, each node $i(i \neq z)$ computes $R_{i}(1)$ and $k_{1}^{\star}$ based on Theorem 3.1 and Eq. (4). Then it broadcasts $R_{i}(1)$ to any node $j$ for which $i \in \mathcal{N}_{j}$. At the beginning of Step 2, each node $i$ knows $R_{j}(1)$ for $j \in \mathcal{N}_{i}$ from Step 1 . Hence, these nodes can compute $k_{2}^{\star}$ using Theorem 3.1 and $R_{i}(2)$ using Eq. (4). At the end of Step 2, node $i$ broadcasts $R_{i}(2)$ to all nodes $j$ with $i \in \mathcal{N}_{j}$, and the procedure repeats until step $D$. At this point, we have computed $R_{i}(D)$ for all nodes, but also $k_{d}^{\star}$ and $R_{i}(d)$ for all $d \in[1, D]$ which is all information needed by nodes to execute their optimal local forwarding policies. As shown next, executing the optimal local forwarding policies for the appropriate delay constraint results in a routing strategy that maximizes the deadlineconstrained reliability.

Theorem 3.2: Letting nodes forward packets according to their optimal local forwarding policies $\Gamma_{k_{d}^{\star}}$ maximizes the probability that the packet generated by the source node arrives at the sink node within the deadline $D$.

The same analysis procedure will be used repeatedly in this paper. We first derive the optimal one-step next-hop decision, and then invoke Theorem 3.2 to justify that we can maximize $R_{i}(D)$ for a given $D$ using these optimal decisions.

\section{B. Solution for information patterns $(b)$ and (c)}

Under information patterns (b) and (c), the true states of outgoing links at the next time slot are no longer available. Since erasure events in the Bernoulli model are independent from one time slot to the next, information about link states at earlier times does not help the scheduling decision. This will lead to simple routing policies that unconditionally attempt to forward packets to the most reliable next-hop node.

For information pattern (a) each node is assumed to know the state of all its outgoing links at the next time slot, so it can postpone the transmission decision if none of its candidate links is in good state. However, under information patterns (b) and (c), this is no longer the case:

Theorem 3.3: The optimal policy under information patterns (b) and (c) does not allow any waiting strategy.

Proof: Consider a node that holds the packet at time $t$ but refrains from making any transmission attempts until time $t+\tau$ with $\tau \geq 1$ when it tries to forward the packet to node $j$. Then, the policy that also tries to transmit to $j$ at time $t$ has higher reliability since there is a non-zero possibility that the transmission is successful, and receiving the packet already at time $t$ will give node $j$ more opportunities to forward the packet, hence a higher reliability.

For any node $i$ that holds a packet with maximum delay constraint $d \in[1, D]$, the probability of delivering the packet to the destination if it forwards the packet to node $j \in \mathcal{N}_{i}$ is

$$
R_{i}(d)=q(i, j) R_{j}(d-1)+p(i, j) R_{i}(d-1) .
$$

The optimal scheduling policy is to transmit on the link $(i, j)$ that maximizes $R_{i}(d)$, i.e. the optimal next hop for node $i$ is

$$
j_{d}^{\star}=\arg \max _{j \in \mathcal{N}_{i}}\left(q(i, j) R_{j}(d-1)+p(i, j) R_{i}(d-1)\right) .
$$

Similarly to information pattern (a), for any $i \in \mathcal{N}^{\prime}$ and a specified deadline $D, R_{i}(D)$ can be calculated using the same distributed dynamic programming scheme with Eq. (7) and Eq. (8). It is optimal too denoted by Theorem 3.2.

Remark: From the optimal policy of unconditionally transmitting to the most reliable next-hop, one might be misled to believe that a shortest-path routing with the appropriate weights would be optimal. However, it is important to note that the optimal path might change when the delay constraint changes. Hence, a series of packets originating from the same source node with the same delay constraint, may follow different paths. One such example is given in Figure 2, where packets are normally forwarded along the more reliable twohop path. However, if a packet has experienced multiple packet losses and remains at the source node when there is only a single slot left until the deadline, the transmission decision will be to try the less reliable direct path.

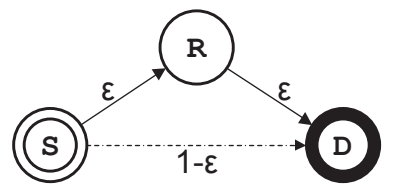

Fig. 2. The longer path consists of two very reliable links. However, a packet with deadline 1 will be transmitted over the unreliable direct path.

\section{REliable ROUTING UNDER Gilbert-Elliot losses}

We are now ready to address the maximum reliability routing problem for the Gilbert-Elliot model, i.e. for arbitrary values of $q_{G}$ and $q_{B}$ in the Markov chain in Figure 1. The channel now has memory, which correlates information and decisions from one time slot to the next. We proceed formally using dynamic programming as in the previous section and find, for information pattern (a) and (b), solutions that despite 
a substantially larger state space, remain tractable for most reasonable industrial network scenarios. For information pattern (c), on the other hand, we will only discuss a special low-complexity scheduling policy.

\section{A. Solution for information pattern (a)}

In the more general Gilbert-Elliot model, the future evolution of the link state is no longer independent of the previous state. Hence, the reliability of keeping the packet at a node $i$ in a specific time slot will now depend on the state of all the outgoing links of node $i$, which complicates the computation of $R_{i}(d)$. To this end, let $\omega \in \Omega_{i}$ be the vector of states of outgoing links at node $i$, and let $\Omega_{i}=\{G, B\}^{\mathcal{N}_{i}}$ be the set of all possible realizations of the link state vector. We use $R_{i}(d \mid \omega)$ to denote the conditional reliability of delivering a packet within a maximal delay $d$ given the state $\omega$ at the next time slot. Since node $i$ does not know the link states of its neighboring nodes, the (unconditional) reliability $R_{j}(d-1)$ of neighbors is used for the routing decision. The optimal decision for node $i$ with a maximum delay constraint $d$, given $R_{j}(d-1), j \in \mathcal{N}_{i}$ and $R_{i}(d-1 \mid \omega) \forall \omega \in \Omega_{i}$, is then as follows: find the highest reliability obtained by forwarding the packet on a link $(i, j)$ in good state and denote this reliability by $R_{j^{\star}}(d-1)$. Then compare it with reliability of keeping the packet at node $i$ :

$$
\sum_{\omega_{n} \in \Omega_{i}} R_{i}\left(d-1 \mid \omega_{n}\right) \operatorname{Pr}\left(\omega_{n} \mid \omega\right),
$$

where $\operatorname{Pr}\left(\omega_{n} \mid \omega\right)$ is the link state transition probability,

$$
\begin{aligned}
& \operatorname{Pr}\left(\omega_{n} \mid \omega\right)= \prod_{m \in G(\omega) \bigcap G\left(\omega_{n}\right)} q_{G}(m) \prod_{n \in G(\omega) \cap B\left(\omega_{n}\right)} p_{G}(n) \\
& \prod_{k \in B(\omega) \bigcap G\left(\omega_{n}\right)} q_{B}(k) \prod_{l \in B(\omega) \bigcap B\left(\omega_{n}\right)} p_{B}(l) .
\end{aligned}
$$

and $G(\omega)$ and $B(\omega)$ is the set of links in good state and bad state, respectively. Execute the best of these two options, which leads to the reliability

$$
R_{i}(d \mid \omega)=\max \left\{R_{j}^{\star}(d-1), \text { Equation }(9)\right\}
$$

The expected forwarding reliability is then

$$
R_{i}(d)=\sum_{\forall \omega \in \Omega_{i}} R_{i}(d \mid \omega) \operatorname{Pr}(\omega),
$$

where $\operatorname{Pr}(\omega)$ is the stationary probability that $\omega$ occurs:

$$
\operatorname{Pr}(\omega)=\prod_{m \in G(\omega)} \pi_{G}(m) \prod_{n \in B(\omega)} \pi_{B}(n) .
$$

Both $R_{i}(D \mid \omega)$ and $R_{i}(D)$ can be computed by applying Eq. (10) and Eq. (11) recursively using the algorithm described in Section III-A starting with the initial values

$$
\begin{aligned}
R_{z}(d) & =1, \quad \forall d=[0, D] ; \\
R_{i}(0)=R_{i}(0 \mid \omega) & =0, \quad \forall i \neq z, \omega \in \Omega_{i} .
\end{aligned}
$$

In each step $d \in[1, D], R_{i}(d)$ and $R_{i}(d \mid \omega) \forall \omega \in \Omega_{i}$ are calculated. $R_{i}(d)$ is then transmitted to all nodes $j$ with $i \in$
$\mathcal{N}_{j}$. Optimality of $R_{i}(D)$ follows by Theorem 3.2 since the optimal local decision is made by each node in each step.

However, contrary to the corresponding results for the Bernoulli model, it is not easy to analytically characterize the set of optimal next-hop nodes. Therefore, we need to iterate over all possible state vectors in $\Omega_{i}$ to determine the optimal decision. Every node needs to evaluate Eq. (9) and Eq. (10) for all $2^{\left|\mathcal{N}_{i}\right|}$ possible link state vectors to compute $R_{i}(d \mid \omega)$, and needs another $2^{\left|\mathcal{N}_{i}\right|}$ steps to compute $R_{i}(d)$ based on Eq. (11). Thus, the computational complexity at each node in each step is $4^{\left|\mathcal{N}_{i}\right|}$. Although the complexity increases exponentially, it remains tractable for most reasonable industrial network scenarios in which each node typically would use a handful (say three to five) of outgoing links.

\section{B. Solution for information pattern $(b)$}

By similar arguments as in Theorem 3.3, the optimal policy under information pattern (b) does not admit any waiting policy, but nodes should always try to transmit on the link that maximizes $R_{i}(d)$. However, the computation of $R_{i}(d)$ becomes more involved, partially since we only know the link state at the previous time slot, and partially because link losses are correlated in time. With some abuse of notation, we now let $R_{i}(d \mid \omega)$ denote the conditional reliability of node $i$ meeting a maximum delay constraint $d$ given that its outgoing links were in state $\omega$ during the previous time slot. Consider a node $i$ that holds a packet with maximum delay constraint $d$ and whose outgoing links were in state $\omega$ at the previous time slot. Assume that the node knows $R_{i}(d-1 \mid \omega) \forall \omega \in \Omega_{i}$ and $R_{j}(d-1)$ for all $j \in \mathcal{N}_{i}$. If the node decides to transmit to node $j \in \mathcal{N}_{i}$, the forwarding reliability becomes

$$
R_{j}(d-1)
$$

if the transmission is successful and

$$
\sum_{\omega_{n} \in B_{j}} R_{i}\left(d-1 \mid \omega_{n}\right) \frac{\operatorname{Pr}\left(\omega_{n} \mid \omega\right)}{p_{i j}(\omega)}
$$

if the transmission fails. Here, $B_{j}$ is the set of link states in which link $(i, j)$ is in bad state. $p_{i j}(\omega)$ is the probability that the link $(i, j)$ is in bad state at the next transmission slot under link state $\omega$, i.e.

$$
p_{i j}(\omega)= \begin{cases}p_{G}(i, j), & \text { if }(i, j) \text { is in good state in } \omega \\ p_{B}(i, j), & \text { otherwise }\end{cases}
$$

Note that $p_{i j}(\omega)$ is also the packet loss probability of link $(i, j)$ at the next transmission slot. Therefore, combining Eq. (12), Eq. (13) and Eq. (14), the packet forwarding reliability of node $i$ with maximum delay constraint $d$ and link status $\omega$, when it uses link $(i, j)$, is

$$
\begin{aligned}
& R_{i j}(d \mid \omega)=\left(1-p_{i j}(\omega)\right) R_{j}(d-1) \\
& \quad+p_{i j}(\omega) \sum_{\omega_{n} \in B_{j}} R_{i}\left(d-1 \mid \omega_{n}\right) \frac{\operatorname{Pr}\left(\omega_{n} \mid \omega\right)}{p_{i j}(\omega)} \\
& =\left(1-p_{i j}(\omega)\right) R_{j}(d-1)+\sum_{\omega_{n} \in B_{j}} R_{i}\left(d-1 \mid \omega_{n}\right) \operatorname{Pr}\left(\omega_{n} \mid \omega\right)
\end{aligned}
$$


The optimal policy is to transmit on the link $\left(i, j_{d}^{\star}\right)$ that maximizes $R_{i j}(d \mid \omega)$, i.e. the optimal next hop for node $i$ is

$$
j_{d}^{\star}=\arg \max _{j \in \mathcal{N}_{i}}\left(R_{i j}(d \mid \omega)\right),
$$

so that

$$
R_{i}(d \mid \omega)=R_{i j_{d}^{\star}}(d \mid \omega) .
$$

Finally the unconditional reliability is computed like Eq. (11)

$$
R_{i}(d)=\sum_{\omega \in \Omega_{i}} R_{i}(d \mid \omega) \operatorname{Pr}(\omega) .
$$

The distributed scheme proposed in Section III-A can also be applied to solve this problem using the initial conditions:

$$
\begin{aligned}
R_{z}(d) & =1, & \forall d=[0, D] ; \\
R_{i}(0)=R_{i}(0 \mid \omega) & =0, & \forall i \neq z, \forall \omega .
\end{aligned}
$$

The solution is optimal by following Theorem 3.2. To understand its computational complexity, note that $R_{i j}(d \mid \omega)$ can be computed in $2^{\left|\mathcal{N}_{i}\right|}$ steps from Eq. (15) and repeated $2^{\left|\mathcal{N}_{i}\right|}$ times of all $\omega$ and $\left|\mathcal{N}_{i}\right|$ times of all $j$. Thus the overall complexity at each node in each step is $\left|\mathcal{N}_{i}\right| \cdot 4^{\left|\mathcal{N}_{i}\right|}$.

\section{Special scheduling in information pattern (c)}

Under information pattern (c) we do not get regular access to all link states, but know the state of one specific link only after we attempt a transmission on that link. Therefore we have to keep track of the conditional reliability for all link configurations, including "good", "bad" and "unknown" state, across $D$ stages, while in information pattern (b) only "good" and "bad" state across one stage is recorded. Although a similar algorithm is still implementable with limited number of outgoing links, its computational complexity increases significantly. Hence, we will highlight a special case that admits a numerically efficient solution by limiting the number of stages that need to be accounted for.

The solution becomes simple when we limit the number of transmission attempts per node to two and consider the maximum reliability routing problem with deadline $2 H_{i}^{\max }$ where $H_{i}^{\max }$ is the depth of node $i$ in the DODAG. Using the same arguments as in Theorem 3.3, information pattern (c) does not admit any waiting policy. Since no information about the link states are available when the node makes its first transmission decision, the optimal choice is to first transmit to node $j \in \mathcal{N}_{i}$ which maximizes

$$
\pi_{G}(i, j) R_{j}
$$

where $R_{j}$ denotes the reliability of node $j$ with deadline $2 H_{j}^{\max }$. If the transmission fails, the conditional reliability of retransmitting to the same node is

$$
q_{B}(i, j) R_{j}
$$

while the reliability via other neighbors is still given by (16). Thus, the retransmission will be scheduled on the same link only if its conditional reliability in Eq. (17) exceeds the unconditional reliability of forwarding across any of its alternative neighbors shown in Eq. (16). Otherwise, the link with the second highest unconditional reliability is scheduled

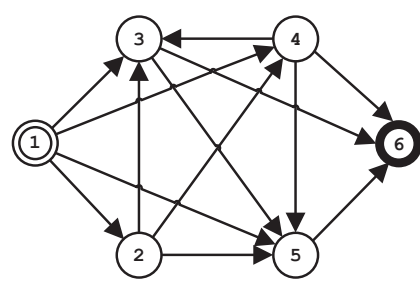

Fig. 3. Example DODAG topology used in Section V

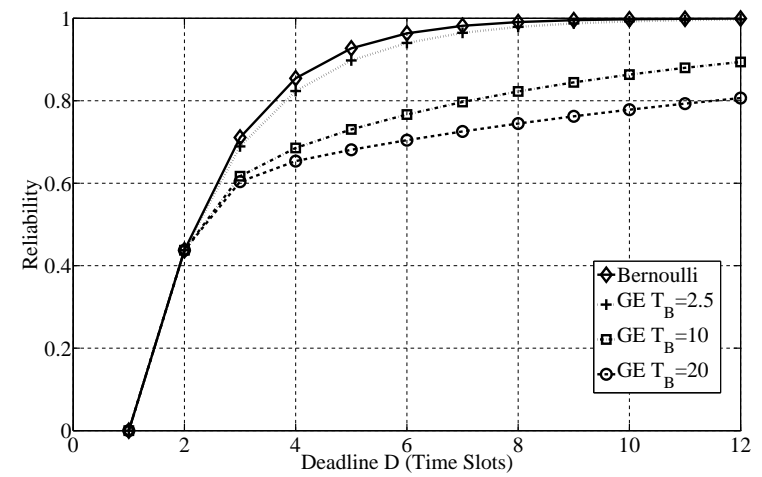

Fig. 4. Deadline-Reliability curves for information pattern (a).

for the second transmission. Let $\left(i, j_{1}\right)$ and $\left(i, j_{2}\right)$ be the links scheduled for the first and second transmission attempt, respectively. The reliability of the proposed policy is then

$$
R_{i}= \begin{cases}\pi_{G}\left(i, j_{1}\right) R_{j_{1}}+\pi_{B}\left(i, j_{1}\right) \pi_{G}\left(i, j_{2}\right) R_{j_{2}}, & \text { if } j_{1} \neq j_{2} ; \\ \pi_{G}\left(i, j_{1}\right) R_{j_{1}}+\pi_{B}\left(i, j_{1}\right) q_{B}\left(i, j_{1}\right) R_{j_{1}}, & \text { otherwise. }\end{cases}
$$

As before, the end-to-end reliability and the optimal scheduling policies can be computed via dynamic programming, traversing the DODAG backwards from the destination node.

\section{NUMERICAL EXAMPLES}

We consider the topology in Figure 3, where a source (node 1) sends packets to a sink (node 6), and analyze the end-to-end reliability for the different loss models and information patterns. We first assume homogeneous links with unconditional loss probability $\pi_{B}=0.5$ for both Bernoulli and GE models. Figure 4 shows the deadlinereliability curves for information pattern (a) under Bernoulli and GE loss models, respectively. The curves display the natural monotonicity of increasing reliability with increasing deadline. Since the minimum hop-count between source and destination is two, the smallest deadline that admits a nonzero reliability is two time slots. The figure also shows that it is harder to maintain high reliability with correlated losses: when a transmission fails, the link is in bad state where it faces a higher conditional loss probability $p_{B}$ than the average loss rate $\pi_{B}$. Furthermore, for fixed $\pi_{B}, p_{B}$ increases with burst length $T_{B}$, which explains why the achievable reliability in Figure 4 decreases with increasing burst length.

Figure 5 shows the value of future information on the achievable reliability. Information pattern (a), where nodes know which outgoing links will be successful when they make scheduling decisions, always outperforms information pattern (b), where nodes cannot be certain scheduled transmissions will be successful. Note that the extra knowledge 


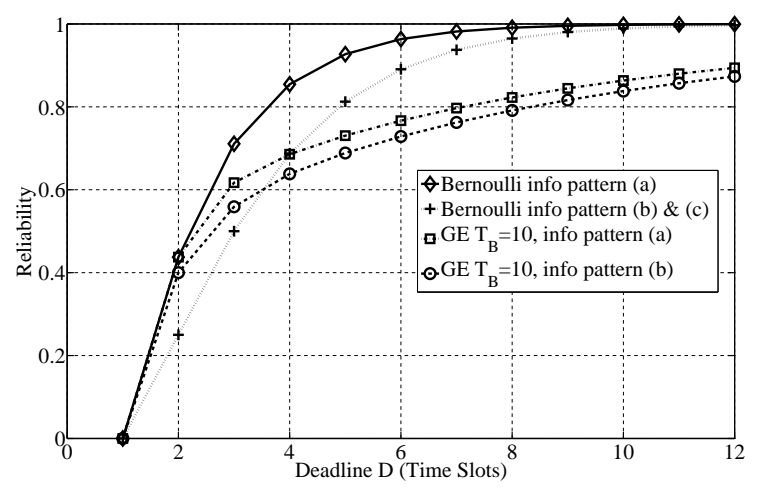

Fig. 5. Deadline-Reliability curves for information patterns (a) and (b).

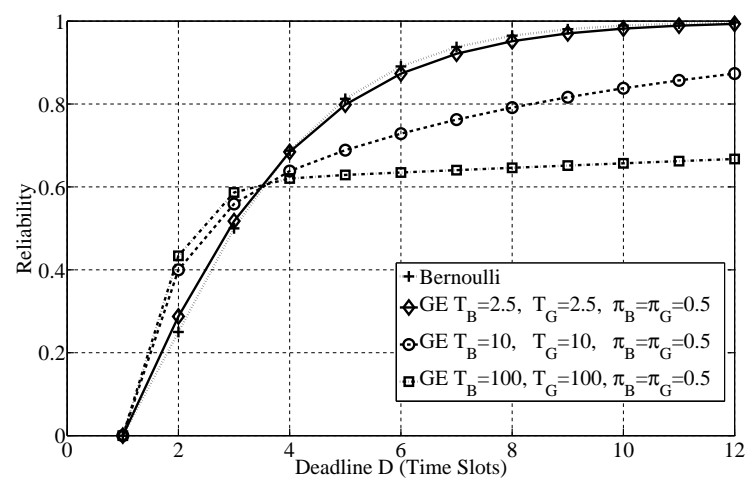

Fig. 6. Deadline-Reliability curves for information pattern (b) with different $T_{B}$ and $T_{G}$ in GE model.

of information pattern (b) compared to information pattern (c) does not help in the Bernoulli model.

Figure 6 considers again homogeneous links with the same (fixed) average behavior in good and bad state, i.e. $\pi_{G}=\pi_{B}=0.5$. The average burst length in good and bad state, i.e. $T_{G}$ and $T_{B}$ respectively, in equation (2) are changed symmetrically to maintain $\pi_{G}=\pi_{B}$. We first notice that the larger the burst length, the better is the reliability for very short deadlines. This follows from Eq. (1) and Eq. (2) by noticing that keeping $\pi_{B}$ fixed and increasing $T_{B}$ yields a larger $T_{G}$. When $T_{G}$ is large, it is very likely that the links that are initially in good state will remain good for a long time, and that routing the packet across these links will be successful at the first attempt. However, this benefit disappears with longer deadlines. The more important observation is that with longer $T_{B}$ it becomes harder to obtain very high reliability even for long deadlines. In this case, if a packet gets blocked at a node where all outgoing links are in bad state, it is likely to suffer a long delay for channel recovery before it can be forwarded.

Finally, Figure 7 shows the average number of times that each link has been used before the packet is dropped or received at the destination under information pattern (b) over $10^{6}$ realizations of Monte Carlo simulation. In this specific example with homogeneous links, the single path $(1 \rightarrow 3 \rightarrow$ 6 ) is optimal when the packet losses are independent and described by the Bernoulli model (see [12] for more details about the performance of single-path routing for deadlineconstrained transmissions in the Bernoulli model). However,

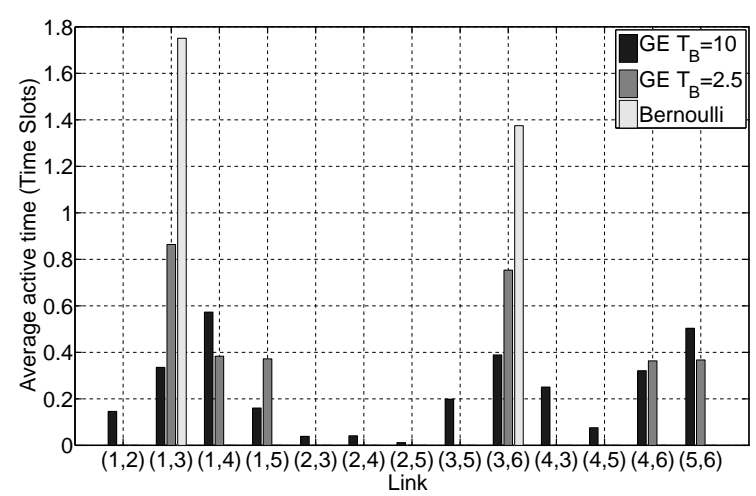

Fig. 7. Average active time under information pattern (b) with $D=4$.

as links become increasingly bursty, it becomes increasingly beneficial to use multi-path routing to avoid links that are in, or likely to be in, bad state. For bursty links described by the GE model with $T_{B}=2.5$, the packets follow three paths $1 \rightarrow 3 \rightarrow 6,1 \rightarrow 4 \rightarrow 6$ and $1 \rightarrow 5 \rightarrow 6$. For longer burst length $T_{B}=10$, all possible paths are used. The comparison of single-path and multi-path routing in the GE model is part of our on-going research.

\section{CONClusions}

We have studied the problem of joint routing and transmission scheduling for maximizing packet delivery probability with a strict deadline constraint in lossy multi-hop networks. A complete solution based on dynamic programming techniques was developed when erasure events on links are independent and follow a Bernoulli process and when links are bursty and losses can be described by the two-state Markov model due to Gilbert and Elliot. Numerical examples illustrated the power of our approach.

\section{REFERENCES}

[1] R. Ogier and V. Rutenburg, "Minimum-expected-delay alternate routing," in INFOCOM '92, vol. 2, 1992, pp. 617-625.

[2] I.-H. Hou, V. Borkar, and P. Kumar, "A theory of QoS for wireless," in INFOCOM 2009, IEEE, April 2009, pp. 486-494.

[3] S. Munir, S. Lin, E. Hoque, S. M. S. Nirjon, J. A. Stankovic, and $\mathrm{K}$. Whitehouse, "Addressing burstiness for reliable communication and latency bound generation in wireless sensor networks," in IPSN '10. Stockholm, Sweden: ACM, 2010, pp. 303-314.

[4] A. Willig, M. Kubisch, C. Hoene, and A. Wolisz, "Measurements of a wireless link in an industrial environment using an IEEE 802.11compliant physical layer," IEEE Transactions on Industrial Electronics, vol. 49, no. 6, pp. 1265-1282, Dec. 2002.

[5] M. H. Alizai, O. Landsiedel, J. A. B. Link, S. Götz, and K. Wehrle, "Bursty traffic over bursty links," in SenSys '09. Berkeley, California: ACM, 2009, pp. 71-84.

[6] D. S. J. De Couto, D. Aguayo, J. Bicket, and R. Morris, "A highthroughput path metric for multi-hop wireless routing," in MobiCom '03, San Diego, CA, USA, 2003, pp. 134-146.

[7] HARTCOMM, WirelessHART Data Sheet, 2007.

[8] ISA, ISA-100.11a-2009 Wireless systems for industrial automation: Process control and related applications, 2009.

[9] T. Winter and P. Thubert, RPL: IPv6 Routing Protocol for Low power and Lossy Networks.

[10] E.N.Gilbert, "Capacity of burst-noise channels," Bell System Technial Journal, vol. 39, pp. 1253-1265, 1960.

[11] E.O.Elliott, "Estimates of error rates for codes on bursty-noise channels," Bell System Technical Journal, vol. 42, pp. 1977-1997, 1963.

[12] P. Soldati, H. Zhang, Z. Zou, and M. Johansson, "Optimal routing and scheduling of deadline-constrained traffic over lossy networks," in IEEE GLOBECOM, Miami FL, USA, Dec. 2010. 